\title{
CHEMICAL ENRICHMENT AND CENTRAL STAR PROPERTIES
}

\author{
C.Y. ZHANG
}

Department of Astronomy, The University of Texas at Austin, Austin, TX 78712

\begin{abstract}
ABSTRAC:T. We have selected a sample of planetary nebulae, for which the core masses are determined using distance-independent parameters (Zhang and Kwok 1992). The chemical abundances of $\mathrm{He}, \mathrm{N}, \mathrm{O}$, and $\mathrm{C}$ are taken from the literature for them. Relationships of the ratios of $\mathrm{He} / \mathrm{H}, \mathrm{N} / \mathrm{O}$, and $\mathrm{C} / \mathrm{O}$ with various stellar parameters of planetary nebulae (PN), such as the core mass, the mass of the core plus the ionized nebular gas, the stellar age and temperature, are examined. It is found that the $\mathrm{N} / \mathrm{O}$ increases with increasing mass, while the $\mathrm{C} / \mathrm{O}$ first increases and then decreases with the core mass. No strong correlation seems to exist between the $\mathrm{He} / \mathrm{H}$ and the core mass. A correlation of the $\mathrm{N} / \mathrm{O}$ and $\mathrm{He} / \mathrm{H}$ with the stellar temperature exists. The current dredge-up theory for the progenitor $\mathrm{A}(\mathrm{GB}$ stars cannot satisfactorily account for these patterns of chemical enrichment in PN. Furthermore, the correlations of the $\mathrm{N} / \mathrm{O}$ and $\mathrm{He} / \mathrm{H}$ with the stellar age and temperature indicate that besides the dredge-ups in the RG and AGB stages, physical piocesses that happen in the planetary nebula stage may also play a role in forming the observed patterns of chemical enrichment in the planetary nebulae.
\end{abstract}

CYZ was supported by the NASA grant NAG 2-67.

\section{References}

Zhang, C.Y., and Kwok, S. 1992, A\&A (in press) 